Article

\title{
High-Performance IGZO Nanowire-Based Field-Effect Transistors with Random-Network Channels by Electrospun PVP Nanofiber Template Transfer
}

\author{
Ki-Woong Park (D) and Won-Ju Cho *(D)
}

check for updates

Citation: Park, K.-W.; Cho, W.-J.

High-Performance IGZO

Nanowire-Based Field-Effect

Transistors with Random-Network

Channels by Electrospun PVP

Nanofiber Template Transfer.

Polymers 2022, 14, 651. https://

doi.org/10.3390/polym14030651

Academic Editor:

Andreea-Teodora Iacob

Received: 20 January 2022

Accepted: 7 February 2022

Published: 8 February 2022

Publisher's Note: MDPI stays neutral with regard to jurisdictional claims in published maps and institutional affiliations.

Copyright: (C) 2022 by the authors. Licensee MDPI, Basel, Switzerland. This article is an open access article distributed under the terms and conditions of the Creative Commons Attribution (CC BY) license (https:/ / creativecommons.org/licenses/by/ $4.0 /)$.
Department of Electronic Materials Engineering, Kwangwoon University, Gwangun-ro 20, Nowon-gu, Seoul 139-701, Korea; wwoong97@naver.com

* Correspondence: chowj@kw.ac.kr

\begin{abstract}
A random network of indium-gallium-zinc oxide (IGZO) nanowires was fabricated by electrospun-polyvinylpyrrolidone (PVP)-nanofiber template transfer. Conventional electrospun nanofibers have been extensively studied owing to their flexibility and inherently high surface-tovolume ratio. However, solution-based IGZO nanofibers have critical issues such as poor electrical properties, reliability, and uniformity. Furthermore, high-temperature calcination, which is essential for vaporizing the polymer matrix, hinders their applications for flexible electronics. Therefore, sputter-based IGZO nanowires were obtained in this study using electrospun PVP nanofibers as an etching mask to overcome the limitations of conventional electrospun IGZO nanofibers. Field-effect transistors (FETs) were fabricated using two types of channels, that is, the nanofiber templatetransferred IGZO nanowires and electrospun IGZO nanofibers. A comparison of the transmittance, adhesion, electrical properties, reliability, and uniformity of these two channels in operation revealed that the nanofiber template-transferred IGZO nanowire FETs demonstrated higher transmittance, stronger substrate adhesion, superior electrical performance, and operational reliability and uniformity compared to the electrospun IGZO nanofiber FETs. The proposed IGZO nanowires fabricated by PVP nanofiber template transfer are expected to be a promising channel structure that overcomes the limitations of conventional electrospun IGZO nanofibers.
\end{abstract}

Keywords: electrospinning; indium-gallium-zinc oxide; random-network nanowire; field-effect transistors; polyvinylpyrrolidone

\section{Introduction}

One-dimensional channel structures, such as nanofibers (NFs), nanowires (NWs), and nanorods, have been actively studied for developing advanced semiconductors; however, they encounter certain physical and technological limitations [1,2]. Owing to their unique properties such as a large surface-to-volume ratio, high transparency, and high flexibility, semiconductor NWs are considered promising building blocks of electronic, photonic, and biochemical sensors [3-6]. In particular, the NW structure prevents the extension of cracks in channels, indicating its suitability for fabricating flexible devices $[7,8]$. Methods used for NW formation include X-ray lithography, deep UV photolithography, vapor-liquidsolid growth, layer-by-layer self-assembly, and scanning probe lithography; however, these techniques can encounter issues owing to their complexity or expensiveness [9-13]. As an effective method for creating NW-like structures, the electrospinning technology enables the facile fabrication of randomly networked NFs and provides various advantages such as low cost, process simplicity, and large-area manufacturing [14,15]. However, electrospun NFs have issues related to poor electrical properties and reliability owing to the high impurity content induced by the solution-based process [16]. Furthermore, hightemperature calcination, which is essential for removing the polymer matrix in NFs, makes 
them unviable for practical flexible electronics applications using thermally vulnerable flexible substrates [16-18].

In this study, nanofiber template-transferred randomly networked indium-galliumzinc oxide (IGZO) NWs are proposed to replace conventional electrospun NF channels. We fabricated two types of nanochannels: 1) directly electrospun IGZO NFs and 2) PVP NF template-transferred IGZO NWs. The random network pattern of an NF template was transferred to a sputter-based IGZO thin film through a wet chemical etching process, resulting in the formation of uniform IGZO NWs with a monolayer structure. Among the numerous candidate NW channel materials, IGZO exhibits excellent electrical and optical properties at low processing temperatures $[19,20]$. Electrospun polyvinylpyrrolidone (PVP) nanofibers were applied as an etching mask to create patterned nanochannels for fieldeffect transistors (FETs). Consequently, the PVP NF template transfer method developed in this study lowered the processing temperature, enabling the formation of IGZO NWs on various substrate materials. The morphology of the channel and the optical and mechanical properties of the fabricated NF-template-transferred IGZO NWs were investigated and compared with those of conventional electrospun IGZO NFs. In addition, FET devices were fabricated using the IGZO NWs and NFs as active channels, and their electrical characteristics and long-term reliability were compared.

\section{Experimental}

\subsection{Preparation of the IGZO Precursor Solution and Electrospinning of IGZO Nanofibers (NFs)}

A PVP precursor solution was prepared by stirring and dissolving PVP powder (100 mg; $\mathrm{M}_{\mathrm{w}} \approx 1,300,000$, Sigma-Aldrich, Saint Louis, MO, USA) in ethanol (1.5 mL; $\geq 99.7 \%$ ) at room temperature. An IGZO precursor solution was prepared by dissolving indium nitrate hydrate $\left[\mathrm{In}\left(\mathrm{NO}_{3}\right)_{3} \cdot x \mathrm{H}_{2} \mathrm{O}\right]$, gallium nitrate hydrate $\left[\mathrm{Ga}\left(\mathrm{NO}_{3}\right)_{3} \cdot x \mathrm{H}_{2} \mathrm{O}\right.$, and zinc acetate hydrate $\left[\mathrm{Zn}\left(\mathrm{CH}_{3} \mathrm{COO}\right)_{2} \cdot 2 \mathrm{H}_{2} \mathrm{O}\right]$ at a molar ratio of 2:1:1 in DMF $(0.5 \mathrm{~mL})$ and stirring at room temperature. Subsequently, an IGZO/PVP precursor solution was prepared by adding the dissolved IGZO solution to the PVP precursor solution. The PVP or IGZO/PVP precursor solution was loaded into a syringe with a metal pinhead, which was fixed to a syringe pump (NE-1000, New Era Pump Systems Inc., Farmingdale, NY, USA). The internal diameter of the pinhead needle was $0.635 \mathrm{~mm}$. A copper plate, which was used as a grounded current collector, was placed at a distance of $20 \mathrm{~cm}$ from the metal pinhead, and the target substrate was fixed to the copper plate. The electrospinning was carried out for $2 \mathrm{~min}$ under a positive voltage of $20 \mathrm{kV}$ applied between the metal pinhead and the copper plate. During NF electrospinning, the flow of the precursor solution was maintained at $0.4 \mathrm{~mL} / \mathrm{h}$, and temperature and relative humidity were maintained at $20{ }^{\circ} \mathrm{C}$ and $20 \%$, respectively. Calcination was performed through furnace annealing at $600{ }^{\circ} \mathrm{C}$ for $30 \mathrm{~min}$ (with a lamp-up time of $30 \mathrm{~min}$ ) in an $\mathrm{O}_{2}$ ambient atmosphere to remove the solvent and impurities from the as-spun IGZO NFs.

\subsection{Formation of IGZO Nanowires (NWs)}

$\mathrm{SiO}_{2} / \mathrm{Si}$ substrates were employed to form the PVP NF template-transfer-type IGZO NWs. A $20 \mathrm{~nm}$-thick IGZO film was deposited using a radiofrequency (RF) magnetron sputtering system with an IGZO target $\left(\mathrm{In}_{2} \mathrm{O}_{3} / \mathrm{Ga}_{2} \mathrm{O}_{3} / \mathrm{ZnO}=4: 2: 4.1 \mathrm{~mol} . \%\right)$. The sputtering was performed at RF power of $100 \mathrm{~W}$, working pressure of $6.0 \mathrm{mTorr}$, and Ar flow rate of $30 \mathrm{sccm}$. Then, PVP NFs were electrospun on the IGZO layer and baked at $200{ }^{\circ} \mathrm{C}$ for $10 \mathrm{~min}$ on a hot plate in air to serve as an etching mask. Subsequently, the IGZO layer was wet-etched in a 30:1 buffered oxide etchant (BOE) using the cured PVP NFs as an etch mask. Consequently, the pattern of the electrospun PVP NF template was transferred onto the IGZO film to form a random network of IGZO nanowires. Finally, the PVP NF template was removed by $\mathrm{O}_{2}$ plasma treatment using a reactive ion etching (RIE) system. Figure 1 illustrates the formation of the IGZO NWs by PVP NF template transfer. The electrospun PVP NFs were formed only a few layers and had large open areas. IGZO exposed by these large open areas was etched in the wet etch process. The diameter of the PVP NFs used as 
the etch mask was approximately $500 \mathrm{~nm}$, which was much larger than the thickness of the IGZO layer. Accordingly, the loss in the horizontal direction in the wet etch process was almost neglected.
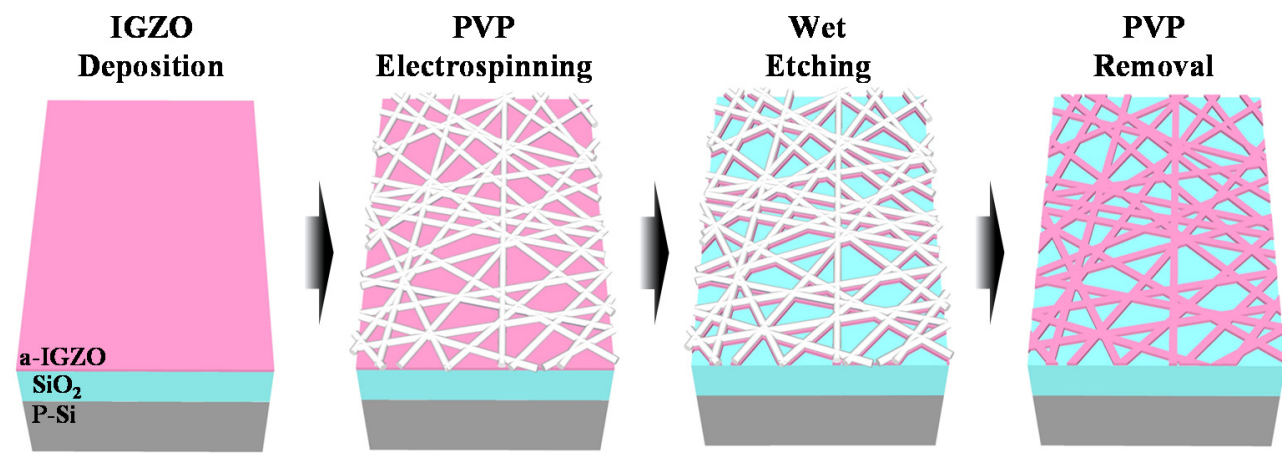

Figure 1. Fabrication of indium-gallium-zinc oxide nanowires (IGZO NWs) by polyvinylpyrrolidone nanofiber (PVP NF) template transfer.

\subsection{Fabrication of IGZO NF- and IGZO NW-Based FETs}

Staggered bottom-gate type FETs were fabricated using the electrospun IGZO NFs and PVP NF template-transfer-type NWs as active channels. A p-type bulk Si (100) substrate with a 100 nm-thick thermally grown $\mathrm{SiO}_{2}$ layer was cleaned using a standard Radio Corporation of America (RCA) cleaning protocol. The IGZO NFs or NWs were formed on the $\mathrm{Si} / \mathrm{SiO}_{2}$ substrates, followed by photolithography and wet etching in 30:1 BOE to define the active regions of the FETs. To eliminate defects and improve the electrical properties of the FETs, the IGZO NF channel was heated at $600{ }^{\circ} \mathrm{C}$ for $30 \mathrm{~min}$ in an $\mathrm{O}_{2}$ ambient, whereas the IGZO NW channel was heated at $250{ }^{\circ} \mathrm{C}$ for $30 \mathrm{~min}$ in $\mathrm{N}_{2}$ ambient. Subsequently, source and drain (S/D) electrodes were formed by depositing a $150 \mathrm{~nm}$-thick Ti film using an electron beam evaporator, followed by a lift-off process. The channel length and width of the fabricated FETs were $100 \mu \mathrm{m}$ and $80 \mu \mathrm{m}$, respectively.

Figure 2 shows a schematic and an optical microscopy image $(300 \times$ magnification) of the fabricated IGZO NW FETs, which corroborated the formation of the NW pattern in the channel region of the FET.
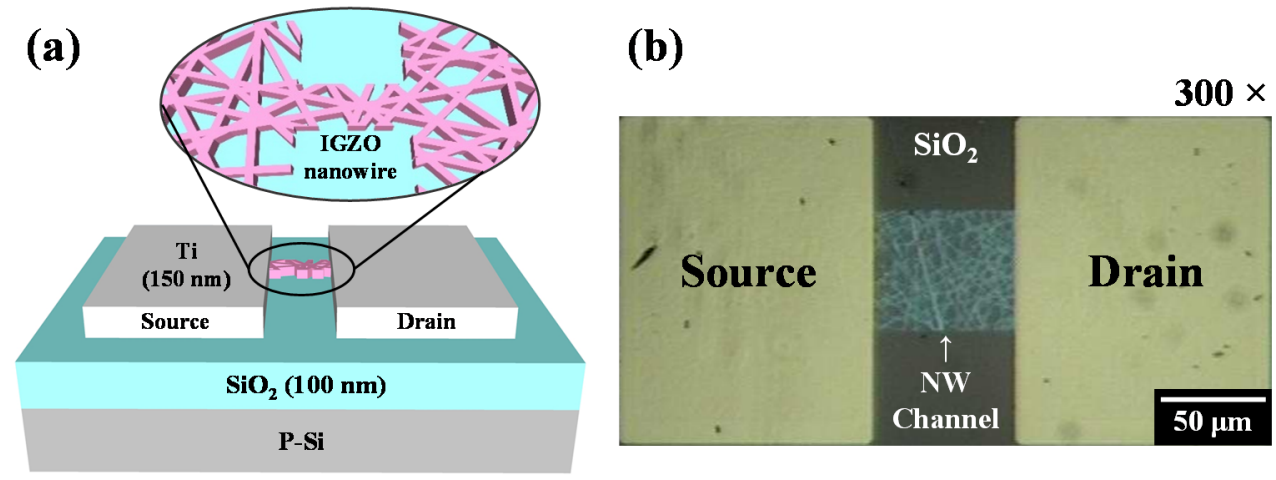

Figure 2. (a) Schematic and (b) optical microscopy image $(300 \times)$ of the IGZO NW-based field-effect transistor (FET) fabricated by PVP NF template transfer.

\subsection{Characterization}

Optical microscopy images of the fabricated IGZO NFs, IGZO NWs, and FETs were acquired using a high-magnification optical microscope (SV-55, SOMETECH, Seoul, Korea). The optical transmittances of the IGZO NWs and NFs were measured using an Agilent 8453 UV-vis spectrophotometer (Hewlett-Packard Co., Palo Alto, CA, USA) in the wavelength range of 190-1100 nm. The surface morphologies of the IGZO NWs and NFs were examined using a DektakXT Bruker stylus profiler (Bruker Corp., Billerica, MA, USA). The electrical 
characteristics of the fabricated IGZO NW- and IGZO NF-channel FETs were evaluated using an Agilent 4156B precision semiconductor parameter analyzer (Agilent Technologies, Wilmington, DE, USA). The measuring devices were placed in a dark box during the electrical measurements to prevent external electrical and optical noise.

\section{Results and Discussion}

Figure $3 \mathrm{a}, \mathrm{b}$ show optical microscopy images (1200× magnification) of the electrospun IGZO NFs and PVP NF template-transferred IGZO NWs, respectively, which indicate that both NFs and NWs had appropriate randomly networked structures.
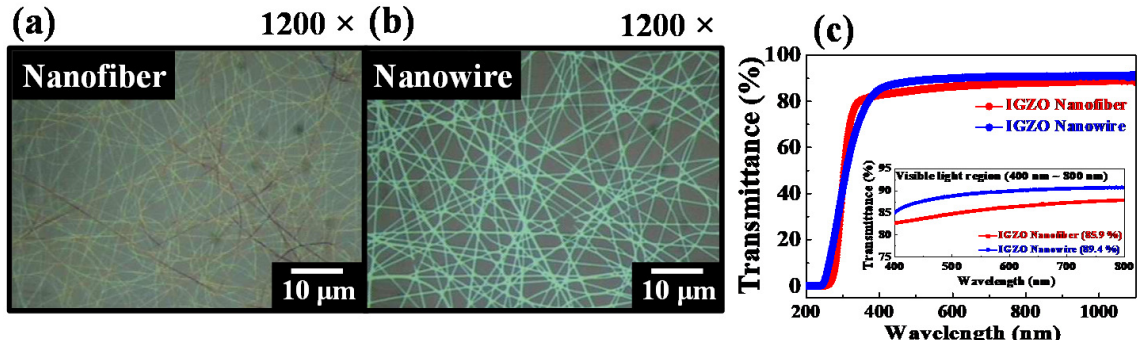

(d)

(e)
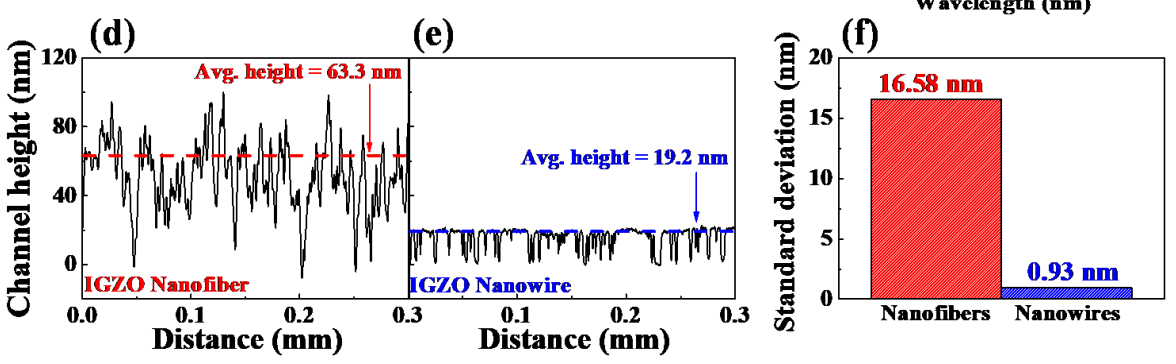

Figure 3. (a,b) optical microscopy images, (c) optical transmittance data (inset shows transmittance data in the visible light region), (d,e) channel height profiles, and (f) standard deviations of the heights of the random networks generated by the electrospun IGZO NFs and the PVP NF template-transferred IGZO NWs.

Figure $3 \mathrm{c}$ shows the optical transmittance data of the IGZO NF and NW random networks fabricated on a glass substrate (7059 glass, Corning Inc., Somervile, MA, USA); the data corresponding to the visible light region $(400-700 \mathrm{~nm})$ are shown in the inset. The average transmittances of the NF and NW random networks were $85.9 \%$ and $89.4 \%$, respectively, indicating that the transmittance of the NFs was lower than that of the NWs. This is because, even in the high-temperature calcination process of the electrospun IGZO $\mathrm{NFs}$, the In, Ga, and Zn components are not completely oxidized, or impurities such as vacancies and polymer matrix still remain [21,22].

Figure 3d,e show the surface height profiles of the IGZO NF and IGZO NW random networks, respectively. The IGZO NFs were randomly laminated during the electrospinning process, resulting in a random network structure with nonuniform thickness and a multilayer channel morphology. The physically stacked multilayer channels of the electrospun IGZO NFs have poor mechanical properties, high contact resistance, and nonuniform electrical properties [23-25]. In contrast, a uniform monolayer channel without a randomly stacked structure was formed by the IGZO NWs via PVP NF template transfer. Therefore, the height of the IGZO NW layer was precisely and uniformly adjusted to the deposition thickness of the sputtering system, and the density (surface coverage) of the NW channels was determined by the electrospinning duration of the PVP NFs. Therefore, the proposed method is advantageous in terms of conveniently fabricating FET devices with the required channel morphology because of the independent control of the height and density of the IGZO NWs.

Figure $3 \mathrm{f}$ shows the standard deviations of the heights of the NF and NW random networks that were extracted from Figure $3 \mathrm{~d}, \mathrm{e}(16.58 \mathrm{~nm}$ and $0.93 \mathrm{~nm}$, respectively). The 
results indicate that the NWs transferred by the PVP NF template had a remarkably uniform thickness compared to the NFs.

The mechanical robustness and substrate adhesion properties of the two prepared random-network nanochannels were evaluated using the tape peeling method [26-28]. Figure 4 shows optical microscopy images and channel height profiles of the random networks of the electrospun IGZO NFs and PVP NF template-transferred IGZO NWs obtained after the tape-peeling experiments. The results suggest that a significant proportion of the NFs in the electrospun IGZO NF random network were peeled off from the $\mathrm{Si} / \mathrm{SiO}_{2}$ substrate upon attaching and detaching $3 \mathrm{M}$ scotch tape (Figure $4 \mathrm{a}$ ). In addition, the average channel height significantly decreased from 69.2 to $27.5 \mathrm{~nm}$, indicating inferior robustness and adhesion to the substrate, which was due to the heterogeneous and randomly stacked structures of the IGZO NFs (Figure 3d). In contrast, the PVP template-transferred IGZO NW random network remained intact after the tape-peeling experiment (Figure $4 \mathrm{~b}$ ), and its average height was maintained at $\sim 20 \mathrm{~nm}$. Therefore, the NF template-transferred IGZO NWs exhibited superior transparency, controllability and uniformity of thickness, and excellent mechanical properties, such as robustness and substrate adhesion.

(a)

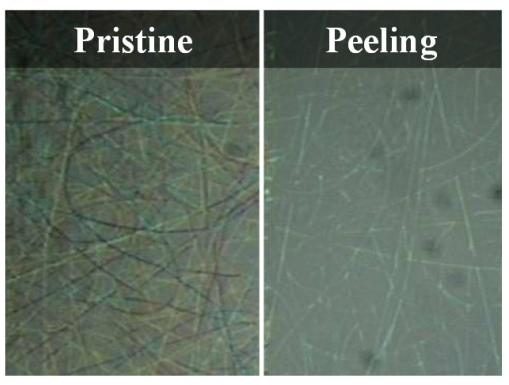

(c)

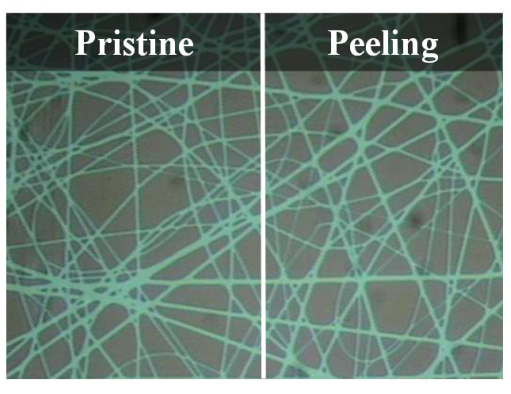

(b)

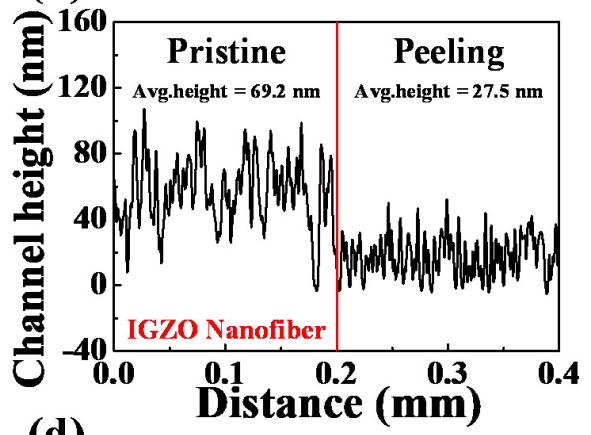

(d)

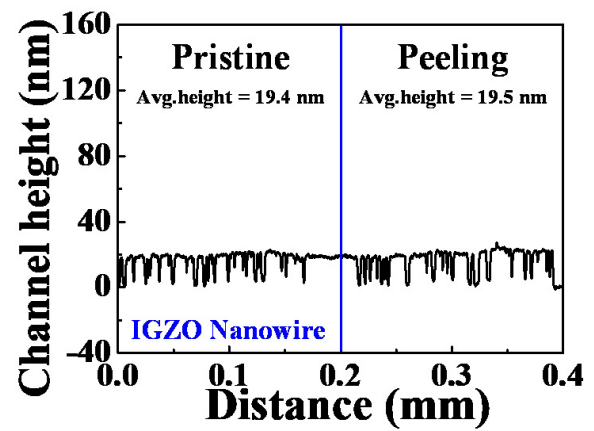

Figure 4. Adhesion tests performed by peeling 3M scotch tape. (a,c) Optical microscopy images and $(\mathbf{b}, \mathbf{d})$ channel height profiles of random networks of the electrospun IGZO NFs $(\mathbf{a}, \mathbf{b})$ and PVP NF template-transferred IGZO NWs (c,d).

Figure 5 shows typical electrical characteristics of the FETs constructed using the electrospun IGZO NFs and PVP template-transferred IGZO NWs. The transfer characteristics $\left(I_{\mathrm{D}}-V_{\mathrm{G}}\right)$ of the NF and NW FETs (Figure 5a) were determined using a double-sweep gate voltage from -20 to $40 \mathrm{~V}$ at a constant drain voltage $\left(V_{\mathrm{D}} ; 10 \mathrm{~V}\right)$, and the output characteristics $\left(I_{\mathrm{D}}-V_{\mathrm{D}}\right.$; Figure $\left.5 \mathrm{~b}\right)$ were determined by varying $V_{\mathrm{D}}(0-20 \mathrm{~V})$ and $\left|V_{\mathrm{G}}-V_{\mathrm{TH}}\right|$ $(0-20 \mathrm{~V})$. The NW FETs were found to exhibit superior electrical performance to that of the NF FETs, with significantly higher on-current, lower leakage current, and a more abrupt change in $I_{\mathrm{D}}$.

To enable a quantitative comparison of the electrical characteristics of the two types of channels, various electrical parameters (averaged values) were extracted (Table 1). The field-effect mobility $\left(\mu_{\mathrm{FE}}\right)$ was calculated using Equation (1), where $C_{\mathrm{ox}}$ is the gate oxide capacitance, $g_{\mathrm{m}}$ is the transconductance, and $V_{\mathrm{D}}$ is the drain voltage from the 
transfer curves [29]. The on/off current ratio $\left(I_{\mathrm{on}} / I_{\text {off }}\right)$ and threshold voltage $\left(V_{\mathrm{TH}}\right)$ were determined from the transfer curves, and the subthreshold swing (SS) was calculated using Equation (2).

$$
\begin{gathered}
\mu_{\mathrm{FE}}=\frac{L}{W C_{\mathrm{ox}} V_{\mathrm{D}}} g_{\mathrm{m}} \\
S S=\frac{d V_{\mathrm{G}}}{d \log I_{\mathrm{D}}}
\end{gathered}
$$

The electrical properties of the PVP NF template-transferred IGZO NW FETs were superior to those of the electrospun IGZO NF FETs owing to their larger $\mu_{\mathrm{FE}}\left(11.81 \mathrm{~cm}^{2} \mathrm{~V}^{-1} \mathrm{~s}^{-1}\right)$, smaller $S S(0.32 \mathrm{mV} / \mathrm{dec})$, and higher $I_{\mathrm{on}} / I_{\mathrm{off}}\left(1.03 \times 10^{9}\right)$. The high $S S$ and low $\mu_{\mathrm{FE}}$ of the electrospun IGZO NF FETs lead to high power consumption and poor frequency response [30]. The hysteresis voltage $\left(V_{\mathrm{H}}\right)$, which is the difference in threshold voltage between the forward and the reverse gate voltage sweeps in the transfer curves, was determined to be $0.45 \mathrm{~V}$ and $12.64 \mathrm{~V}$ for the NW and NF FETs, respectively. Clockwise hysteresis occurred in both devices, which can be primarily attributed to electron trapping at the interface states [31,32]. In particular, the significantly greater $S S$ and $V_{\mathrm{H}}$ in the IGZO NF FETs, despite the use of identical gate dielectric materials, indicate the presence of numerous trap states at the dielectric/channel interface or within the channel. To evaluate the uniformity of the electrical performance, the average value and standard deviation (SD) were also calculated using 10 FETs for each channel type. The relative standard deviation (RSD $=\mathrm{SD} /$ average) of $\mu_{\mathrm{FE}}$ was 0.29 and 0.38 , and that of $S S$ was 0.12 and 0.23 for the NW and NF FETs, respectively. Because the value of $V_{\text {TH }}$ can be zero or negative, the RSD of $V_{\mathrm{TH}}$ cannot enable a meaningful comparison of uniformity of performance [33]. The SD of $V_{\mathrm{TH}}$ was 0.13 and $1.31 \mathrm{~V}$ for the NW and NF FETs, respectively, with the latter being 10 times greater than the former. The PVP template-transferred IGZO NW FETs had lower deviations for $\mu_{\mathrm{FE}}, S S$, and $V_{\mathrm{TH}}$ than the electrospun IGZO NF FETs, indicating more uniform electrical characteristics in the former. Notably, a significant deviation in the $V_{\mathrm{TH}}$ of the IGZO NF FETs adversely affects the operational uniformity, which can lead to malfunctioning of the system.

(a)

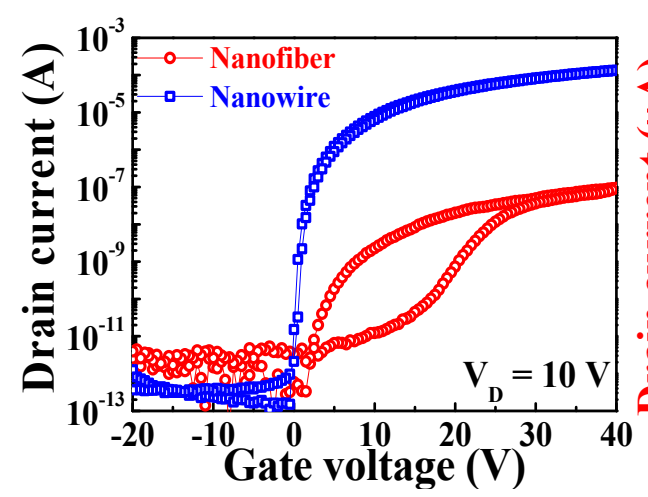

(b)

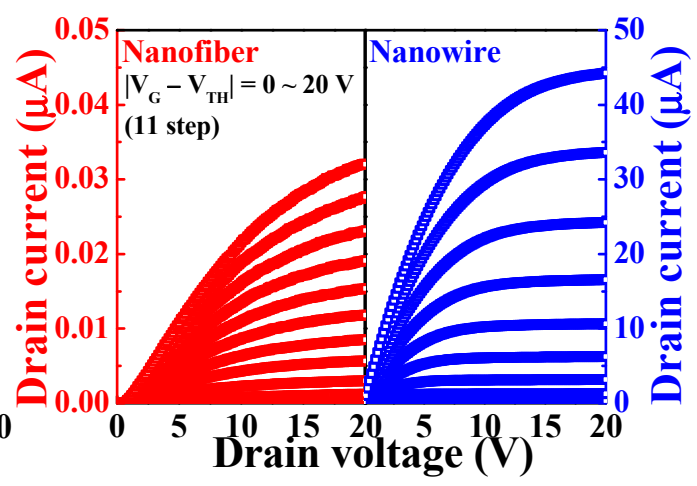

Figure 5. Electrical characteristics of the electrospun IGZO NF FETs and PVP NF-template-transferred IGZO NW FETs: (a) transfer curves $\left(I_{\mathrm{D}}-V_{\mathrm{G}}\right)$ and $(\mathbf{b})$ output curves $\left(I_{\mathrm{D}}-V_{\mathrm{D}}\right)$.

Table 1. Electrical parameters of the electrospun IGZO NF FETs and PVP NF-template-transferred

\begin{tabular}{|c|c|c|c|c|c|c|c|c|}
\hline \multirow{2}{*}{ Channel } & \multicolumn{2}{|c|}{$\mu_{\mathrm{FE}}\left(\mathrm{cm}^{2} \mathrm{~V}^{-1} \mathrm{~s}^{-1}\right)$} & \multicolumn{2}{|c|}{$S S$ (V/dec) } & \multirow{2}{*}{$I_{\mathrm{on}} / I_{\mathrm{off}}$} & \multicolumn{2}{|c|}{$V_{\mathrm{TH}}(\mathrm{V})$} & \multirow{2}{*}{$V_{\mathrm{H}}(\mathrm{V})$} \\
\hline & Avg. & RSD & Avg. & RSD & & Avg. & SD & \\
\hline Nanofibers & 0.16 & 0.38 & 1.62 & 0.23 & $6.28 \times 10^{4}$ & 9.87 & 1.31 & 7.25 \\
\hline Nanowires & 11.81 & 0.29 & 0.32 & 0.12 & $7.39 \times 10^{8}$ & 0.52 & 0.13 & 0.45 \\
\hline
\end{tabular}
IGZO NW FETs. 
Figure 6 shows the time-dependent shifts in the threshold voltage $\left(\Delta V_{\mathrm{TH}}\right)$ of the IGZO NF and NW FETs obtained under positive-bias temperature stress (PBTS) and negative-bias temperature stress (NBTS). These tests are fundamental evaluation methods for determining the stability of FETs based on prolonged gate bias and temperature stressing. For the practical application of the IGZO NFs or NWs, long-term operational stability is crucial for reliable electronic devices. In particular, the evaluation and improvement of device stability are critical for advanced functional materials because of the vulnerability of conventional electrospun NFs to bias stress caused by abundant defects [34]. The PBTS and NBTS conditions were $V_{\mathrm{G}}=V_{\mathrm{TH} 0} \pm 20 \mathrm{~V}$ and $V_{\mathrm{D}}=0 \mathrm{~V}$ at temperatures of 25, 55, and $85^{\circ} \mathrm{C}$ for $10^{3} \mathrm{~s}$, where $V_{\mathrm{TH} 0}$ is the initial threshold voltage of the FETs without gate stress. $\left|\Delta V_{\mathrm{TH}}\right|$, which is the shift of the threshold voltage, was found to increase with increasing stressing temperature and time. This behavior can be explained by the charge-trapping mechanism in oxygen-related trap states (acceptor-like states) or oxygen vacancy-related trap states (donor-like states) [35-37]. When the gate is positively biased, electrons are trapped in the oxygen-related trap states, shifting the threshold voltage in a positive direction. Conversely, a negative gate bias traps holes in the oxygen vacancy-related trap states, shifting the threshold voltage in the negative direction. In addition, because the charge-trapping mechanism is primarily responsible for this shift in threshold voltage, IGZO thin-film transistors (TFTs) with n-type characteristics are generally degraded more severely by the trapping of the majority carriers under the PBTS condition than under the NBTS condition [38]. Moreover, the $\left|\Delta V_{\mathrm{TH}}\right|$ of the NF FETs was considerably higher than that of the NW FETs in both the PBTS and the NBTS tests in the present study. These results indicate the presence of numerous traps at the interface between the gate insulator and the channel and within the channel. In particular, numerous trap states existed in the electrospun IGZO NF channel owing to residual additives. The $\left|\Delta V_{\mathrm{TH}}\right|$ data shown in Figure 6 were adequately fitted using a stretched exponential function, as expressed by Equation (3) [39].

$$
\Delta V_{\mathrm{TH}}=\Delta V_{0}\left\{1-\exp \left[-\left(\frac{t}{\tau}\right)^{\beta}\right]\right\}
$$

where $\Delta V_{0}$ is the $\Delta V_{\mathrm{TH}}$ at infinite stress time, $\tau$ is the charge-trapping time constant, and $\beta$ is the stretched exponent.

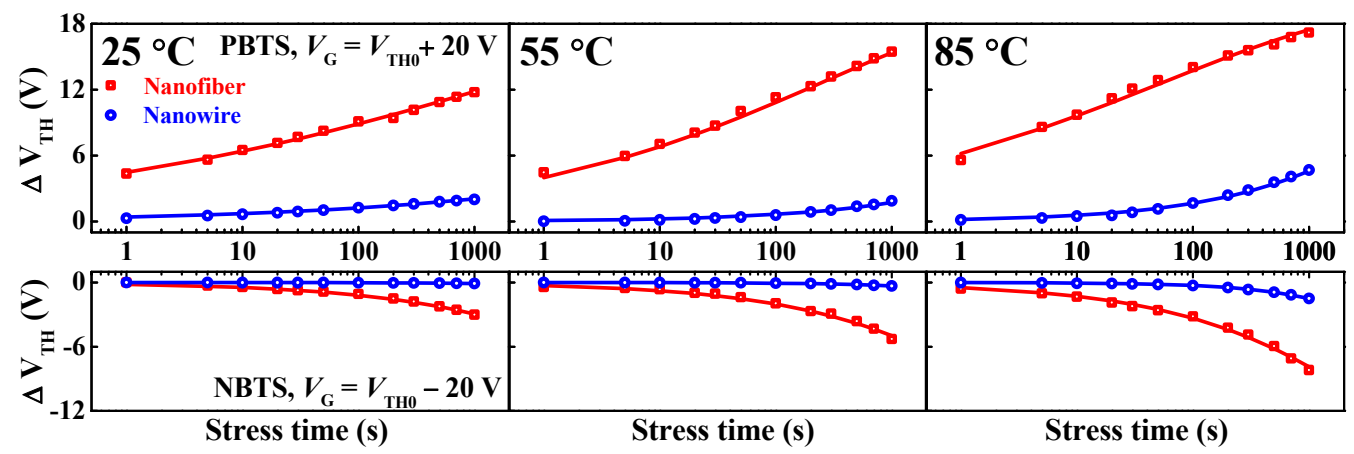

Figure 6. Threshold voltage shifts $\left(\Delta V_{\mathrm{TH}}\right)$ determined by positive-bias temperature stress (PBTS; $V_{\mathrm{G}}=V_{\mathrm{TH} 0}+20 \mathrm{~V}$ ) and negative-bias temperature stress (NBTS; $V_{\mathrm{G}}=V_{\mathrm{TH} 0}-20 \mathrm{~V}$ ) for the electrospun IGZO NF FETs and PVP NF-template-transferred IGZO NW FETs. The dots represent experimental data, and the solid profiles represent the fitting curves obtained using Equation (3).

Figure 7 shows data related to the charge-trapping time constant $(\tau)$, which is the time required for a charge to be captured in the trap states during the PBTS and NBTS tests. As the stressing temperature increased, $\tau$ decreased independently of the gate bias polarity and channel type. In addition, the IGZO NW FETs had higher values of $\tau$ than the IGZO NF FETs in both tests. Notably, the $\tau$ of the NF FETs under PBTS was remarkably small, indicating the occurrence of severe degradation by electron traps in 
the NF FETs. Therefore, the sputtered-film-based IGZO NWs transferred with PVP NF templates enabled the fabrication of FETs with excellent uniform electrical properties and long-term reliability. Consequently, the IGZO NWs designed in this study are anticipated to be effective alternatives to conventional electrospun IGZO NFs.

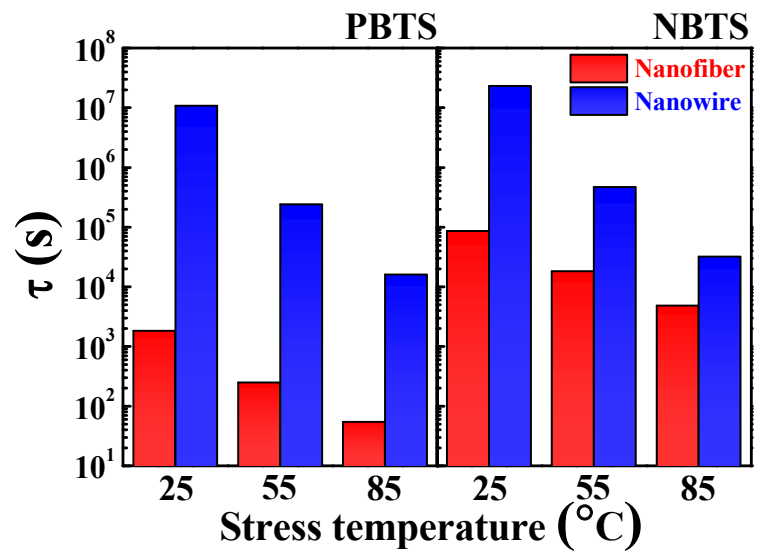

Figure 7. Charge-trapping time constants $(\tau)$ of the electrospun IGZO NF FETs and PVP NF-templatetransferred IGZO NW FETs determined by the PBTS and NBTS tests. The $\tau$ data were extracted by fitting the experimental results to a stretched exponential function.

\section{Conclusions}

Random-network IGZO NWs were fabricated by transferring an electrospun PVP NF template pattern. The PVP NFs, which were used as an etch mask, required a low calcination temperature, and a sputtered IGZO thin film was etched to form randomly networked IGZO NWs. The PVP NF-template-transferred IGZO NWs had a uniform thickness with a monolayer channel, unlike the randomly stacked channels of the electrospun IGZO NFs. The optical transmittance of the IGZO NWs was higher than that of the IGZO NFs because of the low content of impurities such as vacancies and leftover polymer matrix, even at a relatively low process temperature. In addition, the $3 \mathrm{M}$ tape-peeling experiment revealed the superior mechanical robustness and substrate adhesion characteristics of the IGZO NWs. FETs were fabricated using the electrospun IGZO NFs and PVP NF-template-transferred IGZO NWs, and their electrical properties, uniformity, and long-term reliability during operation were evaluated. The PVP NF-template-transferred IGZO NW FETs exhibited outstanding electrical characteristics, such as higher $\mu_{\mathrm{FE}}\left(11.81 \mathrm{~cm}^{2} \mathrm{~V}^{-1} \mathrm{~s}^{-1}\right)$, lower $S S$ $(0.32 \mathrm{~V} / \mathrm{dec})$, larger $I_{\text {on }} / I_{\text {off }}\left(7.39 \times 10^{8}\right)$, and smaller $V_{\mathrm{H}}(0.45 \mathrm{~V})$, than the electrospun IGZO NF FETs. In addition, the IGZO NW FETs exhibited less deviation in these properties, resulting in excellent operational uniformity. To evaluate the reliability, threshold voltage shifts were determined by applying a prolonged gate bias stress under a temperature stress. In both PBTS and NBTS experiments, the IGZO NW FETs showed a lower $\Delta V_{\mathrm{TH}}$ and a longer charge trapping duration $(\tau)$, indicating superior long-term stability. In conclusion, the PVP NF-template-transferred IGZO NWs can presumably be applied in various fields such as flexible electronics, optics, and sensing and replace conventional electrospun IGZO NFs.

Author Contributions: K.-W.P.: conceptualization, formal analysis, methodology, investigation, and writing - original draft. W.-J.C.: conceptualization, methodology, investigation, supervision, validation, and writing-review and editing. All authors have read and agreed to the published version of the manuscript.

Funding: This study was supported by a Research Grant from Kwangwoon University (2021), the Excellent Researcher Support Project of Kwangwoon University (2021), and a National Research Foundation of Korea (NRF) grant funded by the Korean government (MSIT) (No. 2020R1A2C1007586).

Institutional Review Board Statement: Not applicable. 


\section{Informed Consent Statement: Not applicable.}

Data Availability Statement: The data presented in this study are available from the corresponding author upon reasonable request.

Conflicts of Interest: The authors declare no conflict of interest.

\section{References}

1. Zhu, H. Semiconductor nanowire MOSFETs and applications. In Nanowires New Insights; Khan, M., Ed.; IntechOpen: London, UK, 2017; p. 101.

2. Zhang, C.; Yan, Y.; Zhao, Y.S.; Yao, J. Synthesis and applications of organic nanorods, nanowires and nanotubes. Annu. Rep. Prog. Chem. Sect. C Phys. Chem. 2013, 109, 211-239. [CrossRef]

3. Hayden, O.; Agarwal, R.; Lu, W. Semiconductor nanowire devices. Nano Today 2008, 3, 12-22. [CrossRef]

4. Hobbs, R.G.; Petkov, N.; Holmes, J.D. Semiconductor nanowire fabrication by bottom-up and top-down paradigms. Chem. Mater 2012, 24, 1975-1991. [CrossRef]

5. Lin, J.C.; Huang, B.R.; Yang, Y.K. IGZO nanoparticle-modified silicon nanowires as extended-gate field-effect transistor pH sensors. Sens. Actuators B 2013, 184, 27-32. [CrossRef]

6. Larrieu, G.; Han, X.L. Vertical nanowire array-based field effect transistors for ultimate scaling. Nanoscale 2013, 5, 2437-2441 [CrossRef]

7. Kim, Y.G.; Tak, Y.J.; Kim, H.J.; Kim, W.G.; Yoo, H.; Kim, H.J. Facile fabrication of wire-type indium gallium zinc oxide thin-film transistors applicable to ultrasensitive flexible sensors. Sci. Rep. 2018, 8, 5546. [CrossRef]

8. Lee, S.; Jeong, D.; Mativenga, M.; Jang, J. Highly robust bendable oxide thin-film transistors on polyimide substrates via mesh and strip patterning of device layers. Adv. Funct. Mater. 2018, 27, 1700437. [CrossRef]

9. Bourdillon, A.J.; Boothroyd, C.B.; Williams, G.P.; Vladimirsky, Y. Near field X-ray lithography simulations for printing fine bridges. J. Phys. D Appl. Phys. 2003, 36, 2471. [CrossRef]

10. Lin, H.C.; Stehlin, F.; Soppera, O.; Zan, H.W.; Li, C.H.; Wieder, F.; Ponche, A.; Berling, D.; Yeh, B.H.; Wang, K.H. Deep ultraviolet laser direct write for patterning sol-gel InGaZnO semiconducting micro/nanowires and improving field-effect mobility. Sci. Rep. 2015, 5, 10490. [CrossRef]

11. Misra, S.; Yu, L.; Foldyna, M.; i Cabarrocas, P.R. High efficiency and stable hydrogenated amorphous silicon radial junction solar cells built on VLS-grown silicon nanowires. Sol. Energy Mater. Sol. Cells 2013, 118, 90-95. [CrossRef]

12. Zhang, B.; Cui, T. An ultrasensitive and low-cost graphene sensor based on layer-by-layer nano self-assembly. Appl. Phys. Lett. 2011, 98, 073116. [CrossRef]

13. Scappucci, G.; Capellini, G.; Johnston, B.; Klesse, W.M.; Miwa, J.A.; Simmons, M.Y. A complete fabrication route for atomic-scale, donor-based devices in single-crystal germanium. Nano Lett. 2011, 11, 2272-2279. [CrossRef] [PubMed]

14. Yuan, J.J.; Zhu, P.X.; Fukazawa, N.; Jin, R.H. Synthesis of nanofiber-based silica networks mediated by organized poly(ethylene imine): Structure, properties, and mechanism. Adv. Funct. Mater. 2006, 16, 2205-2212. [CrossRef]

15. Wang, B.; Thukral, A.; Xie, Z.; Liu, L.; Zhang, X.; Huang, W.; Yu, X.; Yu, C.; Marks, T.J.; Facchetti, A. Flexible and stretchable metal oxide nanofiber networks for multimodal and monolithically integrated wearable electronics. Nat. Commun. 2020, 11, 2405. [CrossRef]

16. Cho, S.K.; Cho, W.J. Performance enhancement of electrospun IGZO-nanofiber-based field-effect transistors with high-k gate dielectrics through microwave annealing and postcalcination oxygen plasma treatment. Nanomaterials 2020, 10, 1804. [CrossRef]

17. Vander Wal, R.L.; Ticich, T.M.; Curtis, V.E. Substrate-support interactions in metal-catalyzed carbon nanofiber growth. Carbon 2001, 39, 2277-2289. [CrossRef]

18. An, G.H.; Lee, E.H.; Ahn, H.J. Ruthenium and ruthenium oxide nanofiber supports for enhanced activity of platinum electrocatalysts in the methanol oxidation reaction. Phys. Chem. Chem. Phys. 2016, 18, 14859-14866. [CrossRef] [PubMed]

19. Kamiya, T.; Hosono, H. Material characteristics and applications of transparent amorphous oxide semiconductors. NPG Asia Mater. 2010, 2, 15-22. [CrossRef]

20. Fuh, C.S.; Liu, P.T.; Teng, L.F.; Huang, S.W.; Lee, Y.J.; Shieh, H.P.D.; Sze, S.M. Effects of microwave annealing on nitrogenated amorphous In-Ga-Zn-O thin-film transistor for low thermal budget process application. IEEE Electron Device Lett. 2013, 34 , 1157-1159. [CrossRef]

21. Cho, S.K.; Cho, W.J. Microwave-assisted calcination of electrospun indium-gallium-zinc oxide nanofibers for high-performance field-effect transistors. RSC Adv. 2020, 10, 38351-38356. [CrossRef]

22. Munir, M.M.; Widiyandari, H.; Iskandar, F.; Okuyama, K. Patterned indium tin oxide nanofiber films and their electrical and optical performance. Nanotechnology 2008, 19, 375601. [CrossRef]

23. Yarin, A.L.; Koombhongse, S.; Reneker, D.H. Bending instability in electrospinning of nanofibers. J. Appl. Phys. 2001, 89, 3018-3026. [CrossRef]

24. Cui, Y.; Meng, Y.; Wang, Z.; Wang, C.; Liu, G.; Martins, R.; Fortunato, E.; Shan, F. High performance electronic devices based on nanofibers via a crosslinking welding process. Nanoscale 2018, 10, 19427-19434. [CrossRef] [PubMed]

25. Meng, Y.; Lou, K.; Qi, R.; Guo, Z.; Shin, B.; Liu, G.; Shan, F. Nature-inspired capillary-driven welding process for boosting metal-oxide nanofiber electronics. ACS Appl. Mater. Interfaces 2018, 10, 20703-20711. [CrossRef] [PubMed] 
26. Li, J.; Liang, J.; Jian, X.; Hu, W.; Li, J.; Pei, Q. A flexible and transparent thin film heater based on a silver nanowire/heat-resistant polymer composite. Macromol. Mater. Eng. 2014, 299, 1403-1409. [CrossRef]

27. Yin, S.; Zhu, W.; Deng, Y.; Peng, Y.; Shen, S.; Tu, Y. Enhanced electrical conductivity and reliability for flexible copper thin-film electrode by introducing aluminum buffer layer. Mater. Des. 2017, 116, 524-530. [CrossRef]

28. Han, Y.H.; Won, J.Y.; Yoo, H.S.; Kim, J.H.; Choi, R.; Jeong, J.K. High performance metal oxide field-effect transistors with a reverse offset printed Cu source/drain electrode. ACS Appl. Mater. Interfaces 2016, 8, 1156-1163. [CrossRef]

29. Hong, E.K.; Cho, W.J. Effect of microwave annealing on SOI MOSFETs: Post-metal annealing with low thermal budget. Microelectron. Reliab. 2018, 80, 306-311. [CrossRef]

30. Liao, W.; Wei, W.; Tong, Y.; Chim, W.K.; Zhu, C. Low-frequency noise in layered $\mathrm{ReS}_{2}$ field effect transistors on $\mathrm{HfO}_{2}$ and its application for $\mathrm{pH}$ sensing. ACS Appl. Mater. Interfaces 2018, 10, 7248-7255. [CrossRef]

31. Wu, Q.; Wang, J.; Cao, J.; Lu, C.; Yang, G.; Shi, X.; Chuai, X.; Gong, Y.; Su, Y.; Zhao, Y.; et al. Photoelectric plasticity in oxide thin film transistors with tunable synaptic functions. Adv. Electron. Mater. 2018, 4, 1800556. [CrossRef]

32. Hoshino, K.; Hong, D.; Chiang, H.Q.; Wager, J.F. Constant-voltage-bias stress testing of a-IGZO thin-film transistors. IEEE Trans. Electron Devices 2009, 56, 1365-1370. [CrossRef]

33. Chen, M.; Nam, H.; Rokni, H.; Wi, S.; Yoon, J.S.; Chen, P.; Kurabayashi, K.; Lu, W.; Liang, X. Nanoimprint-assisted shear exfoliation (NASE) for producing multilayer $\mathrm{MoS}_{2}$ structures as field-effect transistor channel arrays. ACS Nano 2015, 9, 8773-8785. [CrossRef] [PubMed]

34. Jun, L.; Chen, Q.; Fu, W.; Yang, Y.; Zhu, W.; Zhang, J. Electrospun Yb-doped $\operatorname{In}_{2} \mathrm{O}_{3}$ nanofiber field-effect transistors for highly sensitive ethanol sensors. ACS Appl. Mater. Interfaces 2020, 12, 38425-38434. [CrossRef] [PubMed]

35. Janotti, A.; Van de Walle, C.G. Native point defects in ZnO. Phys. Rev. B 2007, 76, 165202. [CrossRef]

36. Kang, M.S.; Cho, W.J. Improvement of on/off current ratio of amorphous In-Ga-Zn-O thin-film transistor with off-planed source/drain electrodes. J. Nanosci. Nanotechnol. 2019, 19, 1345-1349. [CrossRef]

37. Billah, M.M.; Chowdhury, M.D.H.; Mativenga, M.; Um, J.G.; Mruthyunjaya, R.K.; Heiler, G.N.; Tredwell, T.J.; Jang, J. Analysis of improved performance under negative bias illumination stress of dual gate driving a-IGZO TFT by TCAD simulation. IEEE Electron Device Lett. 2016, 37, 735-738. [CrossRef]

38. Baek, G.; Abe, K.; Kuo, A.; Kumomi, H.; Kanicki, J. Electrical properties and stability of dual-gate coplanar homojunction DC sputtered amorphous indium-gallium-zinc-oxide thin-film transistors and its application to AM-OLEDs. IEEE Trans. Electron Devices 2011, 58, 4344-4353. [CrossRef]

39. Yoon, S.J.; Seong, N.J.; Choi, K.; Shin, W.C.; Yoon, S.M. Investigations on the bias temperature stabilities of oxide thin film transistors using In-Ga-Zn-O channels prepared by atomic layer deposition. RSC Adv. 2018, 8, 25014-25020. [CrossRef] 